PROCEEDINGS OF THE AMERICAN MATHEMATICAL SOCIETY

Volume 124, Number 7, July 1996

\title{
VANISHING OF THE LEADING TERM IN HARISH-CHANDRA'S LOCAL CHARACTER EXPANSION
}

\author{
REID C. HUNTSINGER
}

(Communicated by Roe Goodman)

\begin{abstract}
Harish-Chandra's formula for the character $\Theta_{\pi}$ of an irreducible smooth representation $\pi$ of a reductive $p$-adic group $G$ expresses $\Theta_{\pi}$ near 1 as a linear combination of the Fourier transforms of nilpotent $G$-orbits in the Lie algebra of $G$. In this note, we prove that if $\pi$ is tempered but not in the discrete series, then the coefficient attached to the zero nilpotent orbit vanishes.
\end{abstract}

\section{IntRoduCtion}

Let $\mathbf{G}$ be a connected, reductive algebraic group defined over a $p$-adic field $F$, and let $G=\mathbf{G}(F)$. A smooth irreducible representation of $G$ has a character, which after Harish-Chandra [3, 4] is known to be a locally integrable function on $G$, locally constant on the open subset of regular elements. (See the first two sections of [15] for basic definitions.) Irreducible characters are, of course, basic objects in harmonic analysis on the locally compact group $G$. Since irreducible representations of $G$ are almost always infinite-dimensional, the behavior of their characters near the identity in $G$ is especially interesting. A deep result of Harish-Chandra [4] (and earlier Howe [7] for the general linear group) addresses this topic. Let $\mathcal{O}$ be a nilpotent $G$-orbit in $\mathfrak{g}$; then integration over $\mathcal{O}$ defines a $G$-invariant Radon measure $\mu_{\mathcal{O}}$ on $\mathfrak{g}$ [13]. These are the nilpotent orbital integrals; their Fourier transforms $\hat{\mu}_{\mathcal{O}}$ (taken relative to the additive group of $\mathfrak{g}$ ) are in fact locally integrable functions on $\mathfrak{g}$ which are locally constant on the open subset of regular elements [4]. Let $\pi$ be a smooth irreducible (or just finite length) representation of $G$, and let $\Theta_{\pi}$ be its character. Harish-Chandra's result asserts that there exist complex numbers $c_{\mathcal{O}}(\pi)$ such that

$$
\Theta_{\pi}(\exp X)=\sum_{\mathcal{O}} c_{\mathcal{O}}(\pi) \hat{\mu}_{\mathcal{O}}(X)
$$

where $\mathcal{O}$ runs through nilpotent orbits in $\mathfrak{g}$ and $X$ is a regular element sufficiently close to 0 . We note that (i) the exponential map is defined and invertible in a neighborhood of 0 in $\mathfrak{g}$; and (ii) there are only finitely many nilpotent orbits, so the sum above is finite. (Both statements fail in positive characteristic.)

Little is known in general about the $\hat{\mu}_{\mathcal{O}}$ 's. In case $\mathcal{O}$ is a stable Richardson orbit, the argument of Howe [7] shows that $\hat{\mu}_{\mathcal{O}}$ coincides near 1 with the induced character arising from the trivial representation of the parabolic subgroup corresponding to

Received by the editors September 7, 1994 and, in revised form, November 8, 1994.

1991 Mathematics Subject Classification. Primary 22E50.

Key words and phrases. Character, nilpotent orbit, reductive $p$-adic group. 
$\mathcal{O}$. (See [9] for a generalization.) They can therefore be computed by the formula of Van Dijk [18]. Assem [1] has determined the $\hat{\mu}_{\mathcal{O}}$ 's for $S L_{\ell}(F)$, where $\ell$ is prime.

An important property of the $\hat{\mu}_{\mathcal{O}}$ 's is homogeneity: if $d(\mathcal{O})$ denotes the dimension of the nilpotent orbit $\mathcal{O}$, then we have $\hat{\mu}_{\mathcal{O}}\left(t^{2} X\right)=|t|^{-d(\mathcal{O})} \hat{\mu}_{\mathcal{O}}(X)$ for $X$ regular and $t \in F^{\times}$[4]. Thus the coefficients $c_{\mathcal{O}}(\pi)$ partly determine the asymptotic properties of $\Theta_{\pi}$. (Murnaghan $[10,11]$ has determined these coefficients for supercuspidal representations of certain rank 2 and 3 groups. Mœglin and Waldspurger [9] have generalized a result of Rodier which interprets the coefficients $c_{\mathcal{O}}(\pi)$ for $\mathcal{O}$ regular as the dimension of the space of Whittaker functionals for $\pi$. See also $\S 2$ below.)

We are interested in the coefficient $c_{0}(\pi)$ corresponding to the zero nilpotent orbit. Since $\mu_{0}$ is the Dirac delta at $0 \in \mathfrak{g}$, we have $\hat{\mu}_{0}=1$; thus the term corresponding to the zero nilpotent orbit in (1) is just the constant $c_{0}(\pi)$. The purpose of this note is to prove the following

Theorem. Let $\pi$ be an irreducible tempered representation. Then $c_{0}(\pi)=0$ if $\pi$ is not in the discrete series.

A regular element of $G$ (or $\mathfrak{g}$ ) is called elliptic if its centralizer in $G$ is compact modulo the center of $G$. An irreducible representation $\pi$ is called elliptic if its character does not vanish identically on the set $G^{\text {ell }}$ of elliptic regular elements of $G$. The theorem allows one to distinguish the discrete series characters from other elliptic tempered characters by their behavior on the elliptic set $G^{\text {ell }}$.

For a supercuspidal representation $\pi$, Harish-Chandra showed ([4], Theorem 6) that $c_{0}(\pi)$ equals the formal degree of $\pi$ times a constant depending only on $G$ and the choice of Haar measure on $G$. Rogawski determined the constant in [14]. This formula is known to hold for $\pi$ in the discrete series. We use results of Kazhdan [8] to generalize this formula to arbitrary smooth irreducible $\pi$ (Proposition 1 below, for the case of compact center). The theorem then follows from a simple application of Harish-Chandra's Plancherel formula ( $\$ 5)$.

I would like to thank Paul Sally for introducing me to Harish-Chandra's theory of invariant harmonic analysis and for suggesting the result of this note.

\section{Preliminaries}

We keep the notation of the introduction, but require that the center of $G$ be compact. This allows us to use the results of [8] without modification. This restriction will be removed in $\S 6$.

Let $C_{c}^{\infty}(G)$ be the space of smooth, compactly supported functions on $G$. Fix a Haar measure $d g$ on $G$. For $f \in C_{c}^{\infty}(G)$ let $f^{\vee}(g)=f\left(g^{-1}\right)$. Let $\mathcal{E}(G)$ denote the set of (equivalence classes of) irreducible smooth representations of $G$. Define a pairing $\langle$,$\rangle between irreducible representations and functions:$

$$
\langle\pi, f\rangle=\operatorname{trace} \pi\left(f^{\vee}\right)=\int_{G} \Theta_{\pi}(g) f^{\vee}(g) d g .
$$

Let $\tilde{\pi}$ denote the smooth contragredient of $\pi \in \mathcal{E}(G)$. Then we have $\left\langle\pi, f^{\vee}\right\rangle=\langle\tilde{\pi}, f\rangle$. (In the next section we will attach functions $f \in C_{c}^{\infty}(G)$ to representations $\pi \in \mathcal{E}(G)$ (via the relation (6)). When $\pi$ is supercuspidal, its matrix coefficients lie in $C_{c}^{\infty}(G)$, and we may take $f$ to be a matrix coefficient $f_{\pi}$ with $f_{\pi}(1)=d(\pi)[3$, p. 92-94]. The Schur orthogonality relations [3, p. 4] then insure that $\left\langle\varrho, f_{\pi}\right\rangle=\delta_{\varrho, \pi}$ for any $\varrho \in \mathcal{E}(G)$. Had we defined the pairing $(2)$ to be trace $\pi(f)$, the orthogonality relations would take a slightly less convenient form. See also (7) in §3.) 
The Weyl integration formula [3] allows us to view $\langle$,$\rangle as a pairing between$ characters and orbital integrals. Let $\{T\}$ denote a complete set of representatives of conjugacy classes of Cartan subgroups in $G$, where by a Cartan subgroup we mean the $F$-points of a maximal torus of $\mathbf{G}$ defined over $F$. Then we have

(3)

$$
\int_{G} \Theta_{\pi}(g) f^{\vee}(g) d g=\sum_{\{T\}}\left|W_{T}\right|^{-1} \int_{T^{\mathrm{reg}}}|D(t)| \Theta_{\pi}(t) \int_{G / T} f^{\vee}\left(x t x^{-1}\right) d \dot{x} d t,
$$

where $T^{\text {reg }}$ denotes the regular elements in $T, d t$ is a fixed Haar measure on $T$, $D$ is the usual Weyl discriminant, and $d \dot{x}$ is the quotient measure. If $T$ is elliptic (compact) we choose the measure $d t$ so that the volume of $T$ is 1 .

Orbital integrals have a local expansion, due to Shalika [16], somewhat analogous to that of characters. If $\mathcal{O}$ is a unipotent orbit in $G$, let $\Lambda_{\mathcal{O}}$ denote integration over $\mathcal{O}$. There exist functions $\Gamma_{\mathcal{O}}^{T}$ indexed by unipotent orbits $\mathcal{O}$ and Cartan subgroups $T$ with the following property. Let $f \in C_{c}^{\infty}(G)$; then

$$
\int_{G / T} f\left(x t x^{-1}\right) d \dot{x}=\sum_{\mathcal{O}} \Gamma_{\mathcal{O}}^{T}(t) \Lambda_{\mathcal{O}}(f)
$$

for $t$ a regular element in $T$ sufficiently close to the identity. The functions $\Gamma_{\mathcal{O}}^{T}$ are called Shalika germs. (For a relation between values of the germs $\Gamma_{\mathcal{O}}^{T}$ and the coefficients $c_{\mathcal{O}}$ see [12].)

The germs have homogeneity properties: if $\exp X$ is a regular element of $T$ sufficiently close to the identity, then

$$
\Gamma_{\mathcal{O}}^{T}\left(\exp t^{2} X\right)=|t|^{-d(\mathcal{O})} \Gamma_{\mathcal{O}}^{T}(\exp X)
$$

for $t \in F^{\times}$with $|t| \leq 1$. The germ $\Gamma_{1}^{T}$ associated to the trivial unipotent orbit is constant [4]. $\Gamma_{1}^{T}$ vanishes unless $T$ is elliptic, in which case Rogawski [14] has determined its value. Let $\ell$ denote the semisimple $F$-rank of $G$. Then

$$
\Gamma_{1}^{T}=\frac{(-1)^{\ell}}{d(\mathbf{s t})}
$$

where $T$ is an elliptic Cartan subgroup and $d(\mathbf{s t})$ is the formal degree of the Steinberg representation st with respect to the Haar measure $d g=d \dot{x} d t$.

\section{Some Results of Kazhdan}

We review here some of the results of Kazhdan [8]. Let $R(G)$ be the space of formal $\mathbf{C}$-linear combinations of irreducible representations of $G$. If $P$ is a proper parabolic subgroup of $G$ with Levi factorization $P=M N$, then (unitary) induction defines a map $R(M) \rightarrow R(G)$ (which does not depend on $P$, just $M$ ). Let $R_{I}(G)$ be the subspace spanned by the images of all these maps for proper parabolic subgroups. Let $\bar{R}(G)$ be the quotient $R(G) / R_{I}(G)$. Kazhdan's results imply that the image of $\pi \in \mathcal{E}(G)$ is zero if and only if $\pi$ is not elliptic. (A subtlety, noted first by Herb [6], is that there exist tempered representations which are not elliptic yet are not properly induced.) The Langlands classification shows that $\bar{R}(G)$ is spanned by the classes of irreducible tempered representations.

Let $A(G) \subset C_{c}^{\infty}(G)$ consist of functions whose regular, nonelliptic orbital integrals vanish (such as matrix coefficients of supercuspidal representations [3, p. 92]). Let $J(G)$ be the subspace of functions all of whose regular orbital integrals vanish, including the elliptic ones. Kazhdan shows that $J(G)$ is the space of functions $f$ 
with trace $\pi(f)=0$ for all $\pi \in \mathcal{E}(G)$ (equivalently, for all $\pi$ tempered irreducible), and that $J(G)$ is also the commutator subspace of $C_{c}^{\infty}(G)$. Hence all invariant distributions vanish on $J(G)$. Let $\bar{A}(G)=A(G) / J(G)$.

The pairing (2) extends by linearity to a pairing between $R(G)$ and $C_{c}^{\infty}(G)$. Evidently $J(G)$ is the annihilator of $R(G)$. Since induced characters vanish on the elliptic set (see e.g. [18]), the Weyl integration formula (3) shows that $R_{I}(G)$ annihilates $A(G)$. Thus $\langle$,$\rangle descends to a pairing \langle\rangle:, \bar{R}(G) \times \bar{A}(G) \rightarrow \mathbf{C}$.

The key result of [8] for us is the existence of an isomorphism $\vartheta: \bar{R}(G) \rightarrow \bar{A}(G)$ according to which $\vartheta(\pi)=\bar{f}$ means that the character of $\pi$ is given on the elliptic set by the elliptic orbital integrals of $f$ :

$$
\int_{G} f\left(x \gamma x^{-1}\right) d x=\Theta_{\pi}(\gamma) \text { for } \gamma \in G^{\mathrm{ell}} .
$$

The formula (3) shows that $\vartheta$ is self-adjoint: $\langle\pi, \vartheta(\varrho)\rangle=\langle\varrho, \vartheta(\pi)\rangle$. (In fact this expression is just the elliptic inner product between the characters $\Theta_{\varrho}$ and $\Theta_{\pi}$, for in the formula (3) the nonelliptic Cartan subgroups make no contribution.) We note also that if $\varrho$ is in the discrete series, $\vartheta(\varrho)$ is a pseudo-coefficient for $\varrho$ in the sense that

$$
\langle\pi, \vartheta(\varrho)\rangle= \begin{cases}0 & \text { if } \pi \text { is tempered, irreducible and } \pi \neq \varrho, \\ 1 & \text { if } \pi \cong \varrho .\end{cases}
$$

Indeed, if $f$ is a properly normalized matrix coefficient for $\varrho$, then $\langle\pi, f\rangle$ is given by (7), and by a theorem of Harish-Chandra (cf. [2, Proposition 5]) the regular nonelliptic orbital integrals of $f$ vanish, while on the elliptic set we have the analogue of (6). Thus $f-\vartheta(\varrho)$ is a Schwartz function all of whose regular orbital integrals vanish; by (3) it annihilates all tempered irreducible $\pi$. Then $f$ and $\vartheta(\varrho)$ agree on all tempered irreducible $\pi$, so the relations (7) follow.

\section{A FORMUla FOR THE LEADING TERM}

For $\pi$ in the discrete series, the coefficient $c_{0}(\pi)$ is essentially the formal degree of $\pi$. The formal degree, in turn, is just $f(1)$ if $f$ is a pseudo-coefficient for $\pi$. The following proposition generalizes this relation.

Proposition 1. If $\pi \in \mathcal{E}(G)$ and $\vartheta(\pi)=\bar{f}$, then

$$
c_{0}(\pi)=\frac{(-1)^{\ell}}{d(\mathbf{s t})} f(1) .
$$

Remark. The formal degree $d(\mathbf{s t})$ is computed with respect to the same Haar measure that appears in (6). Changing $d x$ to $c d x$ gives $d(\mathbf{s t}, c d x)=c^{-1} d(\mathbf{s t}, d x)$, and in place of $\bar{f}$ we get $c^{-1} \bar{f}$. Thus the right side is independent of choice of Haar measure, as it should be. Note also that $f(1)$ is independent of the choice of $f$ representing $\bar{f}$.

Proof. In view of (6) we can compare the local character expansion (1) for $\pi$ on the elliptic set with the Shalika germ expansion (4) for the elliptic orbital integrals of $\bar{f}=\vartheta(\pi)$. Let $T$ be any elliptic Cartan subgroup in $G$. We have

$$
\sum c_{\mathcal{O}}(\pi) \hat{\mu}_{\mathcal{O}}(\log \gamma)=\sum \Gamma_{\mathcal{O}}^{T}(\gamma) \Lambda_{\mathcal{O}}(f)
$$

for $\gamma \in T^{\text {reg }}$ sufficiently near 1 . On the left, $\mathcal{O}$ runs through nilpotent orbits in the Lie algebra $\mathfrak{g}$; on the right $\mathcal{O}$ runs through unipotent orbits in $G$. (The exponential 
map gives a bijection between these two sets, preserving the dimension $d(\mathcal{O})$ of the orbit $\mathcal{O}$.)

Set $\gamma=\exp t^{2} X$. We choose $X$ close enough to 0 such that the exponential map is defined for all $t X$ with $|t| \leq 1$, and (8) holds for $0<|t| \leq 1$. Then the homogeneity properties of the $\hat{\mu}_{\mathcal{O}}$ 's and the germs allow us to view equation (8) as an equation between linear combinations of "characters" $|t|^{d}$ of the sub-semigroup $\{t: 0<|t| \leq 1\} \subset F^{\times}$. By linear independence of characters, the coefficients of distinct characters must be equal. Thus

$$
\sum_{\mathcal{O}: d(\mathcal{O})=d} c_{\mathcal{O}}(\pi) \hat{\mu}_{\mathcal{O}}(X)=\sum_{\mathcal{O}: d(\mathcal{O})=d} \Gamma_{\mathcal{O}}^{T}(\exp X) \Lambda_{\mathcal{O}}(f) .
$$

When $d=0$ the only orbits in the sum are the trivial orbits 0 and 1 respectively; hence $c_{0}(\pi)=\Gamma_{1}^{T} f(1)$. Now the proposition reduces to Rogawski's formula (5) for the germ $\Gamma_{1}^{T}$ for the elliptic Cartan subgroup $T$.

Remark. If $\pi$ is square-integrable, we recover the formula of Harish-Chandra and Rogawski using the fact that $f(1)=d(\pi)$. This formula, together with the main theorem, completely determines $c_{0}$ viewed as a linear functional on $\bar{R}(G)$, since, as noted in $\S 3, \bar{R}(G)$ is spanned by tempered irreducible representations.

\section{Vanishing of $f(1)$}

It remains to see that if $\pi$ is tempered but not in the discrete series, then $\bar{f}=\vartheta(\pi)$ vanishes at 1 . For this, we examine the Plancherel formula [5]. Since $f \in A(G)$, its scalar-valued Fourier transform $\hat{f}$ vanishes on induced representations. Thus there is no contribution from proper Levi subgroups, and the Plancherel formula becomes completely explicit:

$$
f(1)=\sum_{\varrho \in \mathcal{E}_{2}(G)} \hat{f}(\varrho) d(\varrho)
$$

where $\mathcal{E}_{2}(G)$ denotes the discrete series representations.

Now $\hat{f}(\varrho)=\operatorname{trace} \varrho(f)$ is just $\langle\pi, \vartheta(\tilde{\varrho})\rangle$ by self-adjointness of $\vartheta$. For $\varrho \in \mathcal{E}_{2}(G)$ and $\pi$ as above, $\vartheta(\tilde{\varrho})$ is a pseudo-coefficient for the discrete series representation $\tilde{\varrho}$; thus by $(7)$ we have $\hat{f}(\varrho)=0$. Thus

Proposition 2. Let $\pi \in \mathcal{E}(G)$ be tempered but not in the discrete series, and let $f$ be a representative of $\bar{f}=\vartheta(\pi)$. Then $\hat{f}(\varrho)=0$ for all $\varrho \in \mathcal{E}_{2}(G)$.

Corollary. With the notation of the proposition, $f(1)=0$.

The theorem (for the case where $G$ has compact center) now follows from this corollary and the formula of Proposition 1.

\section{Conclusion}

To conclude the proof of the theorem, we show how to drop the assumption that $G$ has compact center. Thus let $\mathbf{G}$ be as in the introduction. Let $\mathbf{G}^{\text {der }}$ be the derived subgroup of $\mathbf{G}$, and let $G^{\prime}=\mathbf{G}^{\operatorname{der}}(F)$. Then $G^{\prime}$ has compact, in fact finite, center. Let $\pi$ be an irreducible, tempered, non-square-integrable representation of $G$. By Lemma 2.1 of [17] (valid even in positive characteristic),

$$
\left.\pi\right|_{G^{\prime}}=\pi_{1} \oplus \pi_{2} \oplus \cdots \oplus \pi_{r}
$$


where the $\pi_{i}$ are smooth irreducible representations of $G^{\prime}$. By Proposition 2.7 of [17] each $\pi_{i}$ is tempered and not in the discrete series.

Since a regular elliptic element of $\mathfrak{g}^{\prime}$ is also a regular elliptic element of $\mathfrak{g}$, we can compare the local character expansions of $\pi$ on $G$ and $\left.\pi\right|_{G^{\prime}}$ on $G^{\prime}$ as in the proof of Proposition 1 to see that $c_{0}\left(\left.\pi\right|_{G^{\prime}}\right)=c_{0}(\pi)$. Now the character of $\pi$ restricted to $G^{\prime}$ is the sum of the characters of the $\pi_{i}$; so $c_{0}(\pi)=c_{0}\left(\pi_{1}\right)+c_{0}\left(\pi_{2}\right)+\cdots+c_{0}\left(\pi_{r}\right)=$ $0+\cdots+0=0$. Thus the theorem holds without restriction on the center of $G$.

\section{REFERENCES}

1. M. Assem, The Fourier transform and some character formula for $p$-adic $S L_{\ell}, \ell$ a prime, Amer. J. Math., to appear. CMP 95:05

2. L. Clozel, Invariant harmonic analysis on the Schwartz space of a reductive p-adic group, Harmonic Analysis on Reductive Groups, Bowdoin 1989 (W. Barker \& P. Sally, eds.), Progress in Math., vol. 101, Birkhäuser, Boston, 1991, pp. 101-121. MR 93h:22020

3. Harish-Chandra, Harmonic analysis on reductive p-adic groups, Lecture Notes in Math., vol. 162 (Notes by G. Van Dijk), Springer-Verlag, Berlin, Heidelberg and New York, 1970. MR 54:2889

4. _ Admissible invariant distributions on reductive p-adic groups, Queen's Papers in Pure and Applied Mathematics, vol. 48, Kingston, Ontario, 1978, pp. 281-347. MR 58:28313

5. — The Plancherel formula for reductive -adic groups, Collected Works, vol. IV (ed. V.S. Varadarajan), Springer-Verlag, Berlin, Heidelberg and New York, 1984, pp. 353-367.

6. R. Herb, Elliptic representations for $S p(2 n)$ and $S O(n)$, Pacific J. Math. 161 (1993) 347-358. MR 94i:22040

7. R. Howe, Fourier transform and germs of characters: case of $G L_{n}$ over a p-adic field, Math. Ann. 208 (1974), 305-322. MR 49:7391

8. D. Kazhdan, Cuspidal geometry of p-adic groups, J. Analyse Math. 47 (1986), 1-36. MR 88g:22017

9. C. Møglin and J.L. Waldspurger, Modèles de Whittaker dégénérés pour des groupes p-adiques, Math. Z. 196 (1987), 427-452. MR 89f:22024

10. F. Murnaghan, Asymptotic behavior of supercuspidal characters of p-adic GL(3) and GL(4): the generic unramified case, Pacific J. Math. 148 (1991), 107-130. MR 92a:22023

11. __ Asymptotic behavior of supercuspidal characters of p-adic GSp(4), Comp. Math. 80 (1991), 15-54. MR 92f:22016

12. Characters of supercuspidal representations of classical groups, preprint.

13. Ranga Rao, Orbital integrals on reductive groups, Ann. of Math. 96 (1972), 505-510.

14. J.D. Rogawski, An application of the building to orbital integrals, Comp. Math. 42 (1981), 417-423. MR 83g:22011

15. P.J. Sally, Jr., Some remarks on discrete series characters for reductive p-adic groups, Representations of Lie Groups, Kyoto, Hiroshima, 1986, Adv. Stud. Pure Math. vol. 14, NorthHolland, Amsterdam and New York, 1988, pp. 337-348. MR 91g:22026

16. J.A. Shalika, A theorem on semisimple p-adic groups, Ann. of Math. 95 (1972), 226-242. MR 48:2310

17. M. Tadić, Notes on representations of non-archimedean $S L(n)$, Pacific J. Math. 152 (1992), 375-396. MR 92k:22029

18. G. Van Dijk, Computation of certain induced characters of p-adic groups, Math. Ann. 199 (1972), 229-240.

Department of Mathematics, University of Chicago, Chicago, Illinois 60637

E-mail address: reid@math.uchicago.edu 